Bundesgesundheitsbl $2020 \cdot 63: 267-278$ https://doi.org/10.1007/s00103-020-03098-8 Online publiziert: 7. Februar 2020

(c) Der/die Autor(en) 2020
Nico Dragano' $\cdot$ Marvin Reuter ${ }^{1} \cdot$ Karin Halina Greiser ${ }^{2} \cdot$ Heiko Becher $^{3} \cdot$ Hajo Zeeb ${ }^{4,34} \cdot$ Rafael Mikolajczyk ${ }^{5}$. Alexander Kluttig ${ }^{5}$ Michael Leitzmann ${ }^{6}$. Beate Fischer ${ }^{6} \cdot$ Karl-Heinz Jöckel $^{7}$. Carina Emmel ${ }^{7}$. Gérard Krause ${ }^{8,9}$. Stefanie Castell ${ }^{8} \cdot$ Antje Damms-Machado $^{2} \cdot$ Nadia Obi $^{3} \cdot$ Tamara Schikowski $^{10}$. Oliver Kuss ${ }^{11}$. Wolfgang Hoffmann ${ }^{12} \cdot$ Sabine Schipf ${ }^{12} \cdot$ Tobias Pischon $^{13,14,15,16}$. Lina Jaeschke ${ }^{13}$ - Lilian Krist ${ }^{17}$. Thomas Keil ${ }^{17,18,19}$. Wolfgang Lieb ${ }^{20}$. Bernd Holleczek $^{21,22}$ • Hermann Brenner ${ }^{22,23}$. Kerstin Wirkner ${ }^{24}$.

Markus Loeffler ${ }^{24,25}$. Karin B. Michels ${ }^{26}$. Claus-Werner Franzke ${ }^{26}$. Annette Peters ${ }^{27}$. Jakob Linseisen ${ }^{28,29} \cdot$ Klaus Berger $^{30} \cdot$ Nicole Legath $^{30} \cdot$ Wolfgang Ahrens ${ }^{31,32}$. Thomas Lampert ${ }^{33} \cdot$ Börge Schmidt $^{7}$

' Institut für Medizinische Soziologie, Centre for Health and Society, Medizinische Fakultät, HeinrichHeine-Universität Düsseldorf, Düsseldorf, Deutschland; ${ }^{2}$ Abteilung Epidemiologie von Krebserkrankungen, DKFZ Heidelberg, Heidelberg, Deutschland; ${ }^{3}$ Institut für Medizinische Biometrie und Epidemiologie, Universitätsklinikum Hamburg-Eppendorf, Hamburg, Deutschland; ${ }^{4}$ Abteilung Prävention und Evaluation, Leibniz-Institut für Präventionsforschung und Epidemiologie, BIPS GmbH, Bremen, Deutschland; ${ }^{5}$ Institut für Medizinische Epidemiologie, Biometrie und Informatik, MartinLuther-Universität Halle-Wittenberg, Halle (Saale), Deutschland; ${ }^{6}$ Institut für Epidemiologie und Präventivmedizin, Universität Regensburg, Regensburg, Deutschland; ${ }^{7}$ Institut für Medizinische Informatik, Biometrie und Epidemiologie (IMIBE), Medizinische Fakultät, Universitätsklinikum Essen, Universität Duisburg-Essen, Essen, Deutschland; ${ }^{8}$ Abteilung für Epidemiologie, Helmholtz-Zentrum für Infektionsforschung, Braunschweig, Deutschland; ${ }^{9}$ Medizinische Hochschule Hannover (MHH), Hannover, Deutschland; ${ }^{10}$ IUF - Leibniz Institut für umweltmedizinische Forschung gGmbH, Düsseldorf, Deutschland; "Institut für Biometrie und Epidemiologie, Deutsches Diabetes-Zentrum (DDZ), LeibnizZentrum für Diabetes-Forschung, Heinrich-Heine-Universität Düsseldorf, Düsseldorf, Deutschland; ${ }^{12}$ Institut für Community Medicine, Universitätsmedizin Greifswald, Greifswald, Deutschland;

${ }^{13}$ Forschergruppe Molekulare Epidemiologie, Max-Delbrück-Centrum für Molekulare Medizin in der Helmholtz-Gemeinschaft (MDC), Berlin, Deutschland; ${ }^{14}$ Charité - Universitätsmedizin Berlin, Berlin, Deutschland; ${ }^{15}$ Partnerstandort Berlin, Deutsches Zentrum für Herz-Kreislauf-Forschung (DZHK), Berlin, Deutschland; ${ }^{16} \mathrm{MDC} / \mathrm{BIH}$ Biobank, Max-Delbrück-Centrum für Molekulare Medizin in der HelmholtzGemeinschaft (MDC) und Berlin Institute of Health (BIH), Berlin, Deutschland; ${ }^{17}$ Institut für Sozialmedizin, Epidemiologie und Gesundheitsökonomie, Charité - Universitätsmedizin Berlin, Berlin, Deutschland; ${ }^{18}$ Institut für Klinische Epidemiologie und Biometrie, Universität Würzburg, Würzburg, Deutschland; ${ }^{19}$ Landesinstitut für Gesundheit, Bayerisches Landesamt für Gesundheit und Lebensmittelsicherheit (LGL), Bad Kissingen, Deutschland; ${ }^{20}$ Institut für Epidemiologie, Christian-Albrechts-Universität Kiel, Kiel, Deutschland; ${ }^{21}$ Krebsregister Saarland, Saarbrücken, Deutschland; ${ }^{22}$ Abteilung Klinische Epidemiologie und Alternsforschung, Deutsches Krebsforschungszentrum (DKFZ), Heidelberg, Deutschland; ${ }^{23}$ Abteilung Präventive Onkologie, Deutsches Krebsforschungszentrum (DKFZ) und Nationales Centrum für Tumorerkrankungen (NCT), Heidelberg, Deutschland; ${ }^{24}$ Leipziger Forschungszentrum für Zivilisationserkrankungen, Medizinische Fakultät, Universität Leipzig, Leipzig, Deutschland; ${ }^{25}$ Institut für Medizinische Informatik, Statistik und Epidemiologie (IMISE), Medizinische Fakultät, Universität Leipzig, Leipzig, Deutschland; ${ }^{26}$ Institut für Prävention und Tumorepidemiologie, Universitätsklinikum Freiburg Medizinische Fakultät, Albert-Ludwigs-Universität Freiburg, Freiburg, Deutschland; ${ }^{27}$ Institut für Epidemiologie, Helmholtz Zentrum München, München, Deutschland; ${ }^{28}$ SFG Klinische Epidemiologie, Helmholtz Zentrum München, Neuherberg, Deutschland; ${ }^{29}$ Lehrstuhl für Epidemiologie, UNIKA-T Augsburg, Ludwig-Maximilians-Universität München, Augsburg, Deutschland; ${ }^{30}$ Institut für Epidemiologie und Sozialmedizin, Universität Münster, Münster, Deutschland; ${ }^{31}$ Leibniz-Institut für Präventionsforschung und Epidemiologie, BIPS GmbH, Bremen, Deutschland; ${ }^{32}$ Institut für Statistik Universität Bremen, Bremen, Deutschland; ${ }^{33}$ Abteilung für Epidemiologie und Gesundheitsmonitoring, Robert Koch-Institut, Berlin, Deutschland; ${ }^{34}$ Health Sciences Bremen, Universität Bremen, Bremen, Deutschland

\title{
Soziodemografische und erwerbsbezogene Merkmale in der NAKO Gesundheitsstudie
}




\section{Zusatzmaterial online}

Zusätzliche Informationen sind in der Online-Version dieses Artikels (https://doi. org/10.1007/s00103-020-03098-8) enthalten.

\section{Einleitung}

Das Konzept der NAKO Gesundheitsstudie basiert auf einem biopsychosozialen Verständnis von Gesundheit und Krankheit und es berücksichtigt dementsprechend auch demografische und soziale Merkmale der Teilnehmerinnen und Teilnehmer [1]. Abgesehen davon, dass es zum Standard in epidemiologischen Studien gehört, soziodemografische und erwerbsbezogene Merkmale zu erheben, soll mit der NAKO eine Datenbasis geschaffen werden, die international wegweisende Forschung zu Themen der Sozialepidemiologie ermöglicht.

Dass soziodemografische und erwerbsbezogene Merkmale eine wichtige Rolle bei der Erklärung gesundheitlicher Phänomene spielen, ist unstrittig [2-6]. Alter und Geschlecht sind Kerndeterminanten der Gesundheit, Gleiches gilt für die sozioökonomische Position (üblicherweise als Trias aus Bildung, Einkommen und Beruf verstanden), die familiäre Situation und den Erwerbsstatus. Aus diesem Grund werden soziodemografische Unterschiede in vielen epidemiologischen Untersuchungen berücksichtigt. Darüber hinaus gibt es mit der Sozialepidemiologie eine eigene Forschungsrichtung in der Epidemiologie, die sich explizit mit dieser Art von Einflussfaktoren beschäftigt [7]. Beginnend mit Pionierstudien, wie der britischen Whitehall-Studie, hat diese Forschung eine enge Verbindung insbesondere zwischen der sozioökonomischen Position (folgend als SEP, Socioeconomic Position, abgekürzt) und einer Fülle von Risikofaktoren und gesundheitsrelevanten Verhaltensweisen aufdecken können und gezeigt, dass mit sinkender SEP das Morbiditäts- und Mortalitätsrisiko steigt [7-13].

In den vergangenen zwei Jahrzehnten hat sich in Deutschland das Bewusstsein für die Bedeutung sozialepidemiologischer Ansätze deutlich geschärft, nachdem die Forschung in der Vergan- genheit vor allem durch die angelsächsischen und skandinavischen Länder geprägt war und aus Deutschland nur wenige sowie regional begrenzte Daten zur Forschung beigesteuert wurden [14, 15]. Mittlerweile gibt es hierzulande auch eine wachsende Datenbasis für entsprechende Analysen, sei es in Form von Routinedaten, Bevölkerungsumfragen der Gesundheitsberichterstattung oder verschiedenen Kohortenstudien [14-16]. Aus der Forschung mit diesen Datenquellen ist z. B. bekannt, dass auch in Deutschland erhebliche soziale Ungleichheiten der Gesundheit existieren [17], die sich sowohl entlang von horizontalen Unterschieden (z.B. Geschlecht) als auch von vertikalen Unterschieden (z.B. Bildung oder Einkommen) manifestieren.

Vor diesem Hintergrund ist eine intensive Erforschung der diesen Ungleichheiten zugrunde liegenden $\mathrm{Me}$ chanismen von hohem Interesse. Die bisher verfügbare Datenbasis unterliegt jedoch bestimmten methodischen Einschränkungen. Eine Schwierigkeit ist die oft geringe Tiefe der Differenzierung soziodemografischer Gruppen (etwa in Registerdaten). Diese ist aber notwendig, um (Hoch-)Risikogruppen sicher zu identifizieren und konkrete Zielgruppenbeschreibungen für eine verbesserte Prävention und Versorgung zur Verfügung stellen $\mathrm{zu}$ können. Zusätzlich limitierend wirkt eine vergleichsweise geringe Stichprobengröße mancher Studien, die die Subgruppenbildung weiter erschwert. Zudem konnten in bisherigen Studien soziale Faktoren nur selten direkt in Zusammenhang mit biomedizinischen Indikatoren wie genetischen Informationen, Laborwerten oder bildgebender Diagnostik untersucht werden.

Diese Limitationen können durch große populationsbasierte Kohortenstudien zumindest teilweise ausgeglichen werden. Dies zeigen internationale Beispiele wie die französische CONSTANCES-Studie [18], die niederländische LifeLines-Studie [19], LifeGene aus Schweden [20], CONOR aus Norwegen [21] oder die UK Biobank aus dem Vereinigten Königreich [22]. Die NAKO Gesundheitsstudie folgt diesen Vorbildern und kann so der sozialepide- miologischen Forschung in Deutschland neue Möglichkeiten eröffnen. Die Potenziale der NAKO sind, neben ihrem Längsschnittdesign, die Realisierung einer sehr hohen Fallzahl, die Detailtiefe der Messung von Gesundheit, Krankheit und sozialen Faktoren sowie die Einbeziehung der gesamten in Deutschland lebenden Bevölkerung inklusive jüngerer Altersgruppen ab 20 Jahren [1, 23].

In diesem Beitrag sollen primär die in der NAKO eingesetzten Instrumente zur Messung soziodemografischer und erwerbsbezogener Merkmale vorgestellt werden. Hierzu werden der Prozess der Fragebogenentwicklung und die im Einzelnen ausgewählten Instrumente beschrieben. Ergänzend gibt der Beitrag einen ersten deskriptiven Einblick in die sozialstrukturelle Zusammensetzung der Kohorte und damit auch die konkrete Variablenbildung. Abschließend sollen die eingesetzten Instrumente anhand einer exemplarischen Reproduktion bekannter Zusammenhänge mit dem Body-Mass-Index (BMI) und selbst berichteter Gesundheit validiert werden. Diese Zielgrößen wurden gewählt, weil soziodemografische Unterschiede bei Untergewicht und Adipositas [24, 25] sowie schlechter selbst berichteter Gesundheit [26] in der Forschung gut belegt sind.

\section{Material und Methoden}

\section{Fragebogen und Instrumente}

Der Entwurf des Instruments zur Messung soziodemografischer Angaben einschließlich der sozioökonomischen Position und der Erwerbssituation wurde in den Jahren 2010-2012 von der interdisziplinären "Arbeitsgruppe Fragebogen“ der NAKO Gesundheitsstudie erstellt. Er orientierte sich wesentlich an den „Demographischen Standards“ (DS) des Statistischen Bundesamts in der zum Zeitpunkt der Erstellung gültigen Fassung von 2010 [27]. Diese Standards werden unter anderem beim Mikrozensus eingesetzt und können als Referenzinstrument für Befragungen in Deutschland gelten. Ausgewählt wurden insbesondere Kernvariablen (s. unten). In einzelnen Fällen 
Bundesgesundheitsbl 2020 • 63:267-278 https://doi.org/10.1007/s00103-020-03098-8

(c) Der/die Autor(en) 2020

N. Dragano · M. Reuter · K. H. Greiser · H. Becher · H. Zeeb · R. Mikolajczyk · A. Kluttig · M. Leitzmann · B. Fischer · K.-H. Jöckel · C. Emmel ·

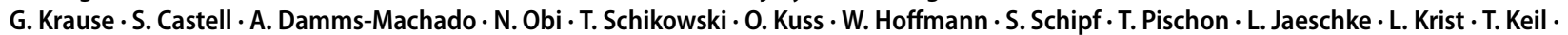
W. Lieb · B. Holleczek · H. Brenner · K. Wirkner · M. Loeffler · K. B. Michels · C.-W. Franzke · A. Peters · J. Linseisen · K. Berger · N. Legath · W. Ahrens · T. Lampert $\cdot$ B. Schmidt

\section{Soziodemografische und erwerbsbezogene Merkmale in der NAKO Gesundheitsstudie}

\section{Zusammenfassung}

Hintergrund. In epidemiologischen Studien ist die standardisierte Erfassung soziodemografischer Merkmale von hoher Bedeutung, da Variablen wie Geschlecht, Alter, Bildung oder Erwerbsstatus wichtige Einflussfaktoren auf Gesundheitschancen und Krankheitsrisiken darstellen. In der NAKO Gesundheitsstudie werden zentrale Faktoren aus diesem Themenbereich berücksichtigt. Ziel der Arbeit. Der Beitrag gibt einen Überblick über den wissenschaftlichen Hintergrund und die konkrete Erhebung soziodemografischer Angaben in der NAKO. Zudem werden die Verteilung einzelner Merkmale sowie Zusammenhänge mit gesundheitsassoziierten Maßen exemplarisch vorgestellt.
Material und Methoden. Anhand der Daten zur Halbzeit der Basiserhebung $(n=101.724)$ wurde die Verteilung soziodemografischer Merkmale dargestellt und Zusammenhänge mit beispielhaft ausgewählten Gesundheitsindikatoren (Body-Mass-Index, selbst berichtete Gesundheit) analysiert, um die Validität der Messung soziodemografischer Angaben zu beurteilen.

Ergebnisse. Das mittlere Alter der Teilnehmenden lag bei 52,0 Jahren ( $S D=12,4) .53,6 \%$ der Teilnehmenden waren Frauen, 54,3\% hatten einen hohen Bildungsabschluss, $60,1 \%$ waren verheiratet zusammenlebend, $72,0 \%$ erwerbstätig und 3,4\% erwerbslos. Bekannte Zusammenhänge zwischen Soziodemografie und Gesundheit konnten reproduziert werden. So waren niedrige Bildung, hohes Alter und Erwerbslosigkeit mit einer erhöhten Häufigkeit von Adipositas und schlechter selbst berichteter Gesundheit assoziiert. Diskussion. Die NAKO Gesundheitsstudie erhebt viele soziodemografische Merkmale. In Kombination mit der Fülle an Gesundheitsdaten und dem Längsschnittdesign ergeben sich so neue Möglichkeiten für die gesundheitswissenschaftliche und sozialepidemiologische Forschung in Deutschland.

\section{Schlüsselwörter}

NAKO Gesundheitsstudie · Sozialepidemiologie - Sozioökonomische Position . Gesundheitliche Ungleichheit · Body-MassIndex

\section{Socio-demographic and employment-related factors in the German National Cohort (GNC; NAKO Gesundheitsstudie)}

\section{Abstract}

Background. In epidemiologic studies, standardised measurement of socio-demographic and employment-related factors is becoming increasingly important, as variables such as gender, age, education or employment status are factors influencing health and disease risks.

Aims. The article gives an overview of the scientific background and assessment of socio-demographic factors in the German National Cohort Study. In addition, the distribution of individual characteristics in the cohort as well as relationships with healthrelated measures are presented by way of example.

Material and methods. The analysis is based on the data of the first half of the baseline survey $(n=101,724)$. On this basis, we present the distribution of key socio-demographic characteristics and analyse relationships with exemplary selected health indicators (body mass index, self-reported health) to assess the validity of socio-demographic data measurements.

Results. On average, study participants were 52.0 years old $(S D=12.4)$. Of the participants, $53.6 \%$ were women, $54.3 \%$ had high education, $60.1 \%$ were married and $72 \%$ were employed while $3.4 \%$ were unemployed. Well-established correlations between sociodemographic factors and health could be reproduced with the German National Cohort data. For example, low education, old age and unemployment were associated with an increased prevalence of obesity and poor selfreported health.

Discussion. The German National Cohort provides a comprehensive measurement of socio-demographic characteristics. Combined with a wide range of health data and the longitudinal measurements available in the future, this opens up new opportunities for health science and social epidemiological research in Germany.

\section{Keywords} German National Cohort - Social epidemiology · Socio-economic position · Social inequality · Body mass index wurde die Abfrage gegenüber dem Original leicht gekürzt oder im Wortlaut etwas verändert, da der Fragebogen der NAKO-Studie aus erhebungsökonomischen Gründen eine gewisse Länge und Komplexität nicht überschreiten durfte. Bei der Erstellung des ersten Entwurfs wurden die Fragen zusätzlich auf Übereinstimmung mit den Instrumenten deutscher Kohortenstudien bzw. Stu- dien der Gesundheitsberichterstattung abgeglichen. Referenzstudien waren beispielsweise CARdiovascular Disease, Living and Ageing in Halle (CARLA), die Studie zur Gesundheit Erwachsener in Deutschland (DEGS), Gesundheit in Deutschland aktuell (GEDA), Heinz Nixdorf Recall (HNR), Kooperative Gesundheitsforschung in der Region Augsburg (KORA), LIFE-Adult des Leipziger
Forschungszentrums für Zivilisationserkrankungen und der Study of Health in Pomerania (SHIP). Einzelne Fragen oder Antwortskalen wurden aus diesen Studien übernommen, wenn die entsprechende Abfrage in den DS entweder nicht vorhanden war (zum Beispiel im Falle der Erfassung von Arbeitslosigkeit) oder wenn die Abfrage in den DS zu komplex 
Tab. 1 Übersicht Demografie, sozioökonomische Position, Erwerbstätigkeit, soziale und berufliche Expositionen

\begin{tabular}{|c|c|c|c|}
\hline Obergruppe & Untergruppen & Details & Quelle $^{a}$ \\
\hline \multirow{2}{*}{$\begin{array}{l}\text { Basis- } \\
\text { demografie }\end{array}$} & Geschlecht & - & DS 2010 \\
\hline & Alter, kontinuierlich & $\begin{array}{l}\text { Berechnet aus Geburtsdatum } \\
\text { (erfragt) und Untersuchungsda- } \\
\text { tum }\end{array}$ & NAKO \\
\hline \multirow[t]{3}{*}{$\begin{array}{l}\text { Familienstand } \\
\text { und Familie }\end{array}$} & Familienstand & - & $\begin{array}{l}\text { DS 2010/ } \\
\text { DEGS } \\
2009\end{array}$ \\
\hline & $\begin{array}{l}\text { Partnerschaft und Zusammen- } \\
\text { leben mit Partner }\end{array}$ & - & $\begin{array}{l}\text { DEGS } \\
2009\end{array}$ \\
\hline & Anzahl leiblicher Kinder & - & NAKO \\
\hline \multirow[t]{2}{*}{$\begin{array}{l}\text { Angaben zum } \\
\text { Haushalt }\end{array}$} & $\begin{array}{l}\text { Wohnsituation (Art der Woh- } \\
\text { nung, z. B. Miete, Eigentum, } \\
\text { Pflegeheim) }\end{array}$ & - & CARLA \\
\hline & $\begin{array}{l}\text { Gesamtzahl Personen im Haus- } \\
\text { halt und Anzahl Personen im } \\
\text { Alter von } 14 \text { Jahren oder älter }\end{array}$ & $\begin{array}{l}\text { Die Zahl der Personen } \leq 14 \text { Jah- } \\
\text { re wird zur Berechnung von } \\
\text { Gewichtungsfaktoren das Äqui- } \\
\text { valenzeinkommens benötigt }\end{array}$ & DS 2010 \\
\hline \multirow[t]{2}{*}{$\begin{array}{l}\text { Schul-und } \\
\text { Ausbildung }\end{array}$} & $\begin{array}{l}\text { Höchster allgemeinbildender } \\
\text { Schulabschluss }\end{array}$ & - & DS 2010 \\
\hline & $\begin{array}{l}\text { Berufliche Ausbildungsab- } \\
\text { schlüsse (Mehrfachnennung) }\end{array}$ & - & DS 2010 \\
\hline Einkommen & $\begin{array}{l}\text { Monatliches Haushaltsnettoein- } \\
\text { kommen }\end{array}$ & Kategoriale Abfrage & DS 2010 \\
\hline \multirow{6}{*}{$\begin{array}{l}\text { Erwerbs- } \\
\text { tätigkeit }\end{array}$} & Aktuelle Erwerbssituation & - & DS 2010 \\
\hline & Wochenarbeitszeit (in Stunden) & Nur erwerbstätige Personen & DS 2010 \\
\hline & $\begin{array}{l}\text { Nichterwerbstätige: aktuelle } \\
\text { Situation }\end{array}$ & - & DS 2010 \\
\hline & $\begin{array}{l}\text { Frühere Erwerbtätigkeit bei } \\
\text { aktuell Nichterwerbstätigen }\end{array}$ & - & DS 2010 \\
\hline & Aktueller oder letzter Beruf & Klartextangaben & DS 2010 \\
\hline & $\begin{array}{l}\text { Berufliche Stellung (Selbst- } \\
\text { ständige, Arbeiter, Angestellte, } \\
\text { Beamte, Auszubildende) }\end{array}$ & Im aktuellen oder letzten Beruf & DS 2010 \\
\hline $\begin{array}{l}\text { Rente/ } \\
\text { Pension/ } \\
\text { Vorruhestand }\end{array}$ & $\begin{array}{l}\text { Berentungsgrund, -jahr, Er- } \\
\text { werbsminderungsrenten Dia- } \\
\text { gnose }\end{array}$ & Nur berentete Personen & NAKO \\
\hline \multirow[t]{2}{*}{$\begin{array}{l}\text { Erwerbs- } \\
\text { losigkeit }\end{array}$} & $\begin{array}{l}\text { Dauer der aktuellen Arbeitslo- } \\
\text { sigkeit }\end{array}$ & $\begin{array}{l}\text { Nur arbeitslos gemeldete Perso- } \\
\text { nen und Personen in Maßnah- } \\
\text { men }\end{array}$ & NAKO \\
\hline & $\begin{array}{l}\text { Frühere Arbeitslosigkeit: jemals } \\
\text { arbeitslos gemeldet, Zahl und } \\
\text { Gesamtdauer der jeweiligen } \\
\text { Perioden }\end{array}$ & - & NAKO \\
\hline $\begin{array}{l}\text { Bildungsstand } \\
\text { Partner }\end{array}$ & $\begin{array}{l}\text { Höchster Schulabschluss und } \\
\text { Ausbildungsabschlüsse Partner }\end{array}$ & - & DS 2010 \\
\hline $\begin{array}{l}\text { Bildung und } \\
\text { Beruf der } \\
\text { Eltern }\end{array}$ & $\begin{array}{l}\text { Höchster Schulabschluss und } \\
\text { berufliche Stellung Vater und } \\
\text { Mutter }\end{array}$ & $\begin{array}{l}\text { Berufliche Stellung zum 13. Le- } \\
\text { bensjahr der Befragten }\end{array}$ & DS 2010 \\
\hline \multicolumn{4}{|c|}{$\begin{array}{l}\text { aDS } 2010=\text { Demographische Standards } 2010 \text { [27], DEGS } 2009=\text { Studie zur Gesundheit Erwachsender } \\
\text { in Deutschland [36], CARLA = CARdiovascular Disease, Living and Ageing in Halle (CARLA) Study } \\
\text { [43], GEDA } 2009=\text { Gesundheit in Deutschland aktuell, GEDA-Studie } 2009 \text { [44], NAKO= eigene } \\
\text { Entwicklung für die NAKO Gesundheitsstudie auf Basis verschiedener Quellen [1] }\end{array}$} \\
\hline
\end{tabular}

erschien. Zudem wurden wenige, meist einfache Fragen neu entwickelt.

Der auf dieser Basis erstellte Fragebogenentwurf wurde externen Expertinnen und Experten, beispielsweise des Robert Koch-Instituts und des Rats für Sozialund Wirtschaftsdaten, zur Kommentierung vorgelegt. Die finale Fassung wurde dann als Teil des Core-Interviews implementiert, ein für alle Studienteilnehmenden obligatorisches, persönliches, computerassistiertes Interview. Die Interviewenden wurden entsprechend geschult und führten die Interviews als Zweiergespräch in eigenen Räumlichkeiten in den Erhebungszentren durch.

- Tab. 1 gibt einen Überblick über die erfassten Themengebiete und verweist auf die Referenzen der eingesetzten Fragen. Durch die Abfrage der Haushaltszusammensetzung ist zudem die Ableitung des Nettoäquivalenzeinkommens [28] möglich, das zusammen mit der Bildung und der beruflichen Stellung ein zentraler Indikator der SEP ist [29]. Anzumerken ist, dass ebenfalls Angaben zum Migrationshintergrund erhoben wurden (siehe Beitrag von Wiessner et al. in diesem Themenheft). Ebenfalls ist darauf hinzuweisen, dass zusätzlich einzelne soziale und berufsbezogene Expositionen erhoben wurden (z. B. soziale Isolation, Arbeitsstress), die hier nicht Gegenstand der Betrachtung sind.

\section{Daten}

Die folgenden Analysen beruhen aufdem Datensatz zur Halbzeit der Basiserhebung der NAKO Gesundheitsstudie. Der Datensatz enthält Informationen zu Teilnehmenden, die zwischen März 2014 und März 2017 in insgesamt 18 Studienzentren die Basisuntersuchung absolviert haben. Allgemeine Angaben zum Studiendesign, zu Ethikvotum und Datenschutz der NAKO sind an anderer Stelle zu finden [1]. Eine Beschreibung der hier verwendeten Stichprobe zur Halbzeit der Basiserhebung und eine detaillierte Beschreibung der Responseraten werden im Beitrag von Schipf et al. in diesem Themenheft präsentiert. Die dort berichtete mittlere Responserate betrug zur Halbzeit $18 \%$, variierte jedoch mit dem Alter. 


\section{Studienpopulation}

Der Datensatz für diese Analyse beinhaltet $n=101.724$ Fälle. Zur Formung der für diese Auswertungen verwendeten Studienpopulation wurden alle nicht vollständig und ordnungsgemäß abgeschlossenen Interviews von den Analysen ausge$\operatorname{schlossen}(n=63)$. Es wurden zudem weitere 10 Fälle ausgeschlossen, die bei der Altersvariable Werte außerhalb des plausiblen Wertebereichs aufwiesen. So ergab sich für die Auswertungen eine Stichprobe von $n=101.651$ Fällen. Die in diese Stichprobe eingeschlossenen Personen waren zum Zeitpunkt der Untersuchung zwischen 20 und 73 Jahre alt. Alle Teilnehmenden waren in Übereinstimmung mit dem Studienprotokoll bei Stichprobenziehung maximal 69 Jahre alt.

\section{Variablen}

Die hier untersuchten Variablen umfassen Informationen zu Alter, Geschlecht, Bildung, Familienstand, Partnerschaft, Wohnsituation, Erwerbsstatus, Beruf, Body-Mass-Index (BMI) und selbst berichteter Gesundheit. Abgeleitete und zusammengefasste Variablen werden im Folgenden beschrieben.

Alter und Bildung. Das Alter des Befragten lag als kontinuierliches Merkmal vor. In Übereinstimmung mit der Routineberichterstattung des Statistischen Bundesamtes und des Robert KochInstituts wurde zusätzlich eine Altersklassifizierung in 10-Jahres-Schritten vorgenommen [30, 31]. Der höchste schulische und berufliche Bildungsabschluss wurde in Übereinstimmung mit den Demographischen Standards [27] erhoben. Die Klassifizierung der Bildungsabschlüsse erfolgte anhand der hierarchischen International Standard Classification of Education von 1997 (ISCED 97; [32]). ISCED ordnet schulische und berufliche Bildungsabschlüsse in einer hierarchischen Skala, die es ermöglicht, Bildungsniveaus international vergleichbar zu machen. Die hier dargestellte ISCED-Skala enthält die folgenden sechs Abstufungen: ISCED 1 (Grundschule), ISCED 2 (Haupt- und Realschule), ISCED 3 (Haupt- und Realschule mit beruflich-betrieblicher oder beruflichschulischer Ausbildung), ISCED 4 (Fachhochschulreife, Abitur, erweiterte Oberschule), ISCED 5 (Fachschulabschluss, Fachakademie oder (Fach-)Hochschulabschluss), ISCED 6 (Promotion). Angemerkt sei, dass Klartextangaben zu sonstigen bzw. ausländischen Bildungsabschlüssen hier unberücksichtigt bleiben $(n=7716)$, da die manuelle Kategorisierung noch nicht abgeschlossen war.

Familie und Partnerschaft. Der rechtliche Familienstand wurde in Übereinstimmung mit den Vorgaben des Statistischen Bundesamtes erhoben und nicht verändert. Um neben ehelichen auch nichteheliche Partnerschaften $\mathrm{zu}$ berücksichtigen, wurde der Familienstand in Kombination mit dem Partnerschaftsstatus zu einer neuen Variable abgeleitet, die angibt, ob Befragte mit einem festen (Ehe-)Partner zusammenleben, mit dem (Ehe-)Partner nicht zusammenleben oder keinen Partner hatten. Die Frage nach der Anzahl der insgesamt im Haushalt lebenden Personen wurde zusammengefasst in: Ein-, Zwei-, Drei- und Vierpersonenhaushalte sowie Haushalte mit 5 Personen und mehr.

Erwerbstätigkeit und Beruf. Zur Bestimmung des Erwerbsstatus wurde auf das Labour-Force-Konzept der International Labour Organisation (ILO) zurückgegriffen [33]. Hier wird unterschieden zwischen Erwerbstätigen, Erwerbslosen und Nichterwerbspersonen. $\mathrm{Zu}$ den Erwerbstätigen zählen Arbeitnehmer, Selbstständige, mithelfende Familienangehörige, Personen in beruflicher Ausbildung, Minijobs, 450Euro-Jobs, Ein-Euro-Jobs sowie Personen in einem temporär inaktiven Arbeitsverhältnis (Mutterschutz, Elternzeit). Erwerbslose sind Personen, die nicht erwerbstätig sind, aber für die Aufnahme einer Erwerbstätigkeit zur Verfügung stehen und aktiv nach einer Arbeit suchen. Nichterwerbspersonen sind Personen, die bereits aus dem Erwerbsleben ausgeschieden sind (Rentner) sowie Schüler und Studenten oder anderweitig dem Arbeitsmarkt nicht zur Verfügung stehende Personen. Die wö- chentliche Arbeitszeit in Stunden wurde als kontinuierliches Merkmal und zur Beschreibung von Voll- und Teilzeit als kategoriale Variable verwendet $(<35$, $\geq 35 \mathrm{~h}$ ). Eine weitere Einteilung der Berufe wird möglich, wenn die Codierung der offenen Berufsabfrage abgeschlossen ist. Für eine erste Einteilung der Berufe wurde jedoch eine Variable abgeleitet, die zwischen Selbstständigen, Arbeitnehmern und Personen in Ausbildung unterscheidet.

Body-Mass-Index (BMI) und selbst berichtete Gesundheit. Der BMI wurde auf Basis der anthropometrischen Messung errechnet und nach Maßgabe der Klassifikation der Weltgesundheitsorganisation (WHO) kategorisiert [34, 35]. Die Messung des BMI ist ausführlich im Beitrag von Fischer et al. in diesem Themenheft beschrieben. Zur Klassifikation von Adipositas wurde eine vierstufige kategoriale Variable berechnet, die zwischen Untergewicht (BMI $\left.<18,5 \mathrm{~kg} / \mathrm{m}^{2}\right)$, Normalgewicht $\left(18,5-24,9 \mathrm{~kg} / \mathrm{m}^{2}\right)$, Präadipositas $\left(25-29,9 \mathrm{~kg} / \mathrm{m}^{2}\right)$ und Adipositas $\left(\geq 30 \mathrm{~kg} / \mathrm{m}^{2}\right)$ unterscheidet. Der selbst berichtete allgemeine Gesundheitszustand wurde durch die Frage: „Wie würden Sie Ihren Gesundheitszustand im Allgemeinen beschreiben?", gemessen. Dabei wurden die Antworten „weniger gut" und "schlecht“ zusammengefasst sowie die Antworten „ausgezeichnet“, "sehr gut" und "gut“.

\section{Statistische Analysen}

In einem ersten Schritt wurde die Verteilung soziodemografischer und erwerbsbezogener Merkmale in der NAKO-Teilpopulation stratifiziert nach Geschlecht und Studienzentrum beschrieben. Absolute sowie relative Häufigkeiten wurden zur Deskription von kategorialen Merkmalen verwendet und Mittelwerte (MW), Standardabweichungen (SD) sowie Mediane und Interquartilsabstände (IQR) zur Beschreibung von kontinuierlichen Merkmalen. Um die Messung soziodemografischer und erwerbsbezogener Merkmale hinsichtlich ihrer Validität zu beurteilen, wurden exemplarisch Zusammenhänge mit dem BMI und selbst berichteter Gesundheit 
Tab. 2 Beschreibung der Teilnehmenden zur Halbzeit der NAKO-Basiserhebung nach soziodemografischen und erwerbsbezogenen Merkmalen

\begin{tabular}{|l|l|l|l|}
$\begin{array}{l}\text { Männer } \\
(n=47.192 ; 46,4 \%)\end{array}$ & $\begin{array}{l}\text { Frauen } \\
(n=54.459 ; 53,6 \%)\end{array}$ & $\begin{array}{l}\text { Gesamt } \\
(n=101.651 ; \\
100 \%)\end{array}$ \\
\hline$n /(M W) \quad \% /(S D)$ & $n /(M W) \quad \% /(S D)$ & $n /(M W) \quad \% /(S D)$
\end{tabular}

Alter in Jahren (fehlende Werte $n=0$ )

(MW, SD)

$(52,5)$

$(12,3)$

$(51,5)$

$(12,4)$

$(52,0)$

Altersgruppen (fehlende Werte $n=0$ )

20-29 Jahre

3029

30-39 Jahre

4348

40-49 Jahre

9837

6,4

50-59 Jahre

13.391

9,2

4047

\section{7,4}

5240

9,6

12.195

22,4

7,0

60-69 Jahre

$15.045 \quad 31,9$

15.970

29,3

$15.580 \quad 28,6$

$1542 \quad 3,3$

70-74 Jahre

$47.192 \quad 100,0$

1427

2,6

$54.459 \quad 100,0$

Bildung (ISCED97; fehlende Werte $n=9,234)^{\text {a }}$

1 Grundschule

$198 \quad 0,5$

2 Haupt- und Realschule

712

1,7

3 Ausbildung

$11.715 \quad 27,2$

4 (Fach-)Hochschulreife

4753

5 Fachschul-

$23.111 \quad 53,7$

\section{6}

1506

0,6

14.364

3,0

8672

29,1

17,6

22.815

46,2

1745

6 Promotion

$2520 \quad 5,9$

Gesamt

$43.009 \quad 100,0$

Familienstand (fehlende Werte $n=25$ )

\begin{tabular}{|c|c|c|c|c|c|c|}
\hline 6 Promotion & 2520 & 5,9 & 1745 & 3,5 & 4265 & 4,6 \\
\hline Gesamt & 43.009 & 100,0 & 49.408 & 100,0 & 92.417 & 100,0 \\
\hline \multicolumn{7}{|c|}{ Familienstand (fehlende Werte $n=25$ ) } \\
\hline Ledig & 11.096 & 23,5 & 12.244 & 22,5 & 23.340 & 23,0 \\
\hline $\begin{array}{l}\text { Verheiratet zusammenle- } \\
\text { bend }\end{array}$ & 30.106 & 63,8 & 30.997 & 56,9 & 61.103 & 60,1 \\
\hline $\begin{array}{l}\text { Verheiratet getrennt } \\
\text { lebend }\end{array}$ & 822 & 1,7 & 1093 & 2,0 & 1915 & 1,9 \\
\hline Geschieden & 4444 & 9,4 & 7291 & 13,4 & 11.735 & 11,5 \\
\hline Verwitwet & 712 & 1,5 & 2821 & 5,2 & 3533 & 3,5 \\
\hline Gesamt & 47.180 & 100,0 & 54.446 & 100,0 & 101.626 & 100,0 \\
\hline \multicolumn{7}{|c|}{ Partnerschaft (fehlende Werten $=139$ ) } \\
\hline $\begin{array}{l}\text { Mit (Ehe-)Partner zusam- } \\
\text { menlebend }\end{array}$ & 35.513 & 75,3 & 36.929 & 67,9 & 72.442 & 71,4 \\
\hline $\begin{array}{l}\text { Mit (Ehe-)Partner nicht } \\
\text { zusammenlebend }\end{array}$ & 4371 & 9,3 & 5384 & 9,9 & 9755 & 9,6 \\
\hline Ohne Partner & 7250 & 15,4 & 12.065 & 22,2 & 19.315 & 19,0 \\
\hline Gesamt & 47.134 & 100,0 & 54.378 & 100,0 & 101.512 & 100,0 \\
\hline \multicolumn{7}{|c|}{ Anzahl leiblicher Kinder (fehlende Werten = 19) } \\
\hline (MW, SD) & $(1,4)$ & $(1,2)$ & $(1,4)$ & $(1,1)$ & $(1,4)$ & $(1,1)$ \\
\hline \multicolumn{7}{|c|}{ Haushaltsgröße (fehlende Werte $n=39$ ) } \\
\hline Einpersonenhaushalt & 8436 & 17,9 & 11.677 & 21,4 & 20.113 & 19,8 \\
\hline Haushalt mit 2 Personen & 22.411 & 47,5 & 26.089 & 47,9 & 48.500 & 47,7 \\
\hline Haushalt mit 3 Personen & 7863 & 16,7 & 8516 & 15,6 & 16.379 & 16,1 \\
\hline Haushalt mit 4 Personen & 6306 & 13,4 & 6169 & 11,3 & 12.475 & 12,3 \\
\hline $\begin{array}{l}\text { Haushalt mit } 5 \text { Personen } \\
\text { oder mehr }\end{array}$ & 2154 & 4,6 & 1991 & 3,7 & 4145 & 4,1 \\
\hline Gesamt & 47.170 & 100,0 & 54.442 & 100,0 & 101.612 & 100,0 \\
\hline
\end{tabular}

Gesamt analysiert. Hierfür wurde die Häufigkeit von Untergewicht, Adipositas und schlechter selbst berichteter Gesundheit nach Geschlecht, Alter, Bildung, Familienstand, Partnerschaft, Erwerbsstatus, Umfang der Erwerbstätigkeit und Berufsstand deskriptiv dargestellt. Abschließend wurde exemplarisch der Zusammenhang zwischen Bildung und den Gesundheitsvariablen adjustiert für Alter und Studienzentrum, getrennt für Männer und Frauen, mithilfe von Marginsplots dargestellt. Alle Analysen wurden mit Stata $15.1 \mathrm{MP}$ durchgeführt (StataCorp LLC, College Station, TX, USA).

\section{Ergebnisse}

\section{Beschreibung der Studien- population}

- Tab. 2 beschreibt die Studienpopulation nach soziodemografischen und erwerbsbezogenen Merkmalen. Der Anteil an Frauen war höher als der der Männer (53,6\% vs. $46,4 \%)$ und das mittlere Alter lag bei 52,0 Jahren $(\mathrm{SD}=12,4)$. Es zeigte sich, dass Männer im Durchschnitt ein Jahr älter waren als Frauen (52,5 vs. 51,5 Jahre). $54,3 \%$ der Teilnehmerinnen und Teilnehmer hatten einen höheren Bildungsabschluss (beispielsweise Fachschul-, Fachhochschul- oder Hochschulausbildung inklusive Promotion). Dabei war der Anteil bei Männern höher als bei Frauen (59,6\% vs. 49,7\%). Die meisten Befragten waren verheiratet zusammenlebend (60,1\%). Der Anteil Verwitweter war bei den Frauen mehr als dreimal höher als bei den Männern (5,2\% vs. 1,5\%). 19,8\% der Studienteilnehmenden lebten in einem Einpersonenhaushalt. Auch hier war der Anteil bei den Frauen höher (21,4\% vs. $17,9 \%)$. In der Studienpopulation waren des Weiteren 72,0\% der Befragten zum Zeitpunkt der Untersuchung erwerbstätig, 3,4\% erwerbslos und 24,6\% waren Nichterwerbspersonen. Ohne Nichterwerbspersonen lag der Anteil an Erwerbslosen bei 4,5\%. Unter den Erwerbstätigen betrug die durchschnittliche wöchentliche Arbeitszeit 34,1 h $(\mathrm{SD}=12,6)$. Frauen waren erwartungsgemäß häufiger in Teilzeit beschäftigt als 


\begin{tabular}{|c|c|c|c|c|c|c|}
\hline & \multicolumn{2}{|c|}{$\begin{array}{l}\text { Männer } \\
(n=47.192 ; 46,4 \%)\end{array}$} & \multicolumn{2}{|c|}{$\begin{array}{l}\text { Frauen } \\
(n=54.459 ; 53,6 \%)\end{array}$} & \multicolumn{2}{|c|}{$\begin{array}{l}\text { Gesamt } \\
(n=101.651 ; \\
100 \%)\end{array}$} \\
\hline & $n /(M W)$ & $\% /(S D)$ & $n /(M W)$ & $\% /(S D)$ & $n /(M W)$ & $\% /(S D)$ \\
\hline \multicolumn{7}{|c|}{ Erwerbsstatus (fehlende Werte $n=683)^{a}$} \\
\hline Erwerbstätig & 34.235 & 73,0 & 38.453 & 71,1 & 72.688 & 72,0 \\
\hline Erwerbslos & 1904 & 4,1 & 1530 & 2,8 & 3434 & 3,4 \\
\hline Nichterwerbsperson & 10.740 & 22,9 & 14.106 & 26,1 & 24.846 & 24,6 \\
\hline Gesamt & 46.879 & 100,0 & 54.089 & 100,0 & 100.968 & 100,0 \\
\hline \multicolumn{7}{|c|}{ Wöchentliche Arbeitszeit (fehlende Werte $n=505$ ) } \\
\hline In Stunden (MW, SD) & $(38,06)$ & $(12,00)$ & $(30,61)$ & $(12,01)$ & $(34,14)$ & $(12,56)$ \\
\hline In Stunden (Median, IQR) & $(40)$ & (3) & (35) & (19) & (39) & $(10)$ \\
\hline \multicolumn{7}{|c|}{ Umfang der Erwerbstätigkeit (fehlende Werte $n=505)^{b}$} \\
\hline Vollzeit ( $\geq 35 \mathrm{~h} /$ Woche) & 27.907 & 82,7 & 19.045 & 50,6 & 46.952 & 65,8 \\
\hline Teilzeit ( $<35 \mathrm{~h} /$ Woche) & 5852 & 17,3 & 18.579 & 49,4 & 24.431 & 34,2 \\
\hline Gesamt & 33.759 & 100,0 & 37.624 & 100,0 & 71.383 & 100,0 \\
\hline \multicolumn{7}{|c|}{ Berufsstand (fehlende Werte $n=301)^{c}$} \\
\hline Selbstständig & 7766 & 16,7 & 5285 & 10,0 & 13.051 & 13,2 \\
\hline Angestellt & 38.393 & 82,8 & 47.289 & 89,4 & 85.682 & 86,3 \\
\hline Ausbildung & 212 & 0,5 & 299 & 0,6 & 511 & 0,5 \\
\hline Gesamt & 46.371 & 100,0 & 52.873 & 100,0 & 99.244 & 100,0 \\
\hline \multicolumn{7}{|c|}{$\begin{array}{l}\text { IQR Interquartilsabstand, ISCED97 International Standard Classification of Education von 1997, } \\
\text { MW Mittelwert, SD Standardabweichung } \\
\text { aHohe Zahl an fehlenden Werten aufgrund noch uncodierter Angaben } \\
\text { bNur Erwerbstätige (ohne betriebliche Ausbildung) } \\
\text { 'Frage nur gestellt, falls jemals erwerbstätig. Falls zurzeit nicht erwerbstätig betraf die Frage den } \\
\text { letzten Beruf }\end{array}$} \\
\hline
\end{tabular}

Männer (49,4\% vs. $17,3 \%)$ und häufiger nichterwerbstätig. Die Mehrheit der Befragten war aktuell oder zuletzt in einem Angestelltenverhältnis beschäftigt $(86,3 \%), 13,2 \%$ waren selbstständig und - bedingt durch die Altersgrenze von 20 Jahren - nur 0,5\% in Ausbildung.

- Abb. 1 zeigt die Verteilung ausgewählter Merkmale in den einzelnen Studienzentren. Generell variierten sozialstrukturelle Merkmale zwischen den Standorten. So lag der Frauenanteil zwischen 50,8\% (Münster) und 57,2\% (Bremen). Das mittlere Alter reichte von 48,3 Jahren (Bremen) bis 54,8 Jahre (Berlin Süd). Der Anteil an Teilnehmenden mit einem hohen Bildungsabschluss war am geringsten in Augsburg (41,9\%) und am höchsten in den Berliner Studienzentren (jeweils $>63 \%$ ). Der Anteil zusammenlebender Verheirateter lag zwischen 47,1\% (Berlin Mitte) und 71,3\% (Augsburg). Die Zahl der Einpersonenhaushalte war erwartungsgemäß in Regionen höher, die einen geringeren in Essen. und selbst berichteter Gesundheit bis schlechte allgemeine Gesundheit. selbst berichteter Gesundheit unter Teilnehmenden mit niedriger Bildung sowie bei erwerbslosen Personen. Dagegen waren Vollzeitbeschäftigung sowie berufliche Selbstständigkeit mit einer besseren Gesundheit assoziiert. Eine (Ehe-)Partnerschaft war zudem mit einer geringeren Häufigkeit von Übergewicht und Adipositas assoziiert. Partnerlose berichteten zudem häufiger von einer schlechten oder weniger guten Gesundheit. Dies kann teilweise durch ein höheres Durchschnittsalter bei Partnerlosen erklärt werden.

- Abb. 2 zeigt die Häufigkeit der vier Gesundheitsvariablen entlang der sechs hierarchischen Bildungsstufen adjustiert für Alter und Studienzentrum. Wie zu erkennen ist, war für Männer und Frauen geringe Bildung mit einer höheren Häufigkeit von Adipositas und schlechter allgemeiner Gesundheit assoziiert. Dagegen fanden wir einen umgekehrten Bildungsgradienten für Untergewicht bei Frauen mit erhöhten Anteilen von Untergewicht bei Frauen mit höherer Bildung. Insgesamt fanden wir die erwarteten $\mathrm{Zu}$ sammenhänge zwischen soziodemografischen und gesundheitsbezogenen Variablen.

\section{Diskussion} aufwiesen. Die Erwerbslosenquote lag zwischen 2,0\% in Regensburg und 6,7\%

\section{Soziodemografie und Gesundheit: exemplarische Analysen zu BMI}

Die Verteilung der exemplarisch untersuchten gesundheitsbezogenen Merkmale ist im Online-Zusatzmaterial (Tab. S1) sowie in ausführlicher Form im Beitrag von Fischer et al. zur Anthropometrie in diesem Themenheft dargestellt. In der Teilstichprobe fanden wir Untergewicht bei $1 \%$ der Probanden und Adipositas bei $22,4 \%$. Weiter berichteten $12,1 \%$ der Studienteilnehmenden eine weniger gute

- Tab. 3 zeigt die Häufigkeit gesundheitsbezogener Merkmale nach ausgewählten Faktoren. Generell fanden wir einen sozialen Gradienten mit höheren Anteilen an Adipositas und schlechter
Die NAKO Gesundheitsstudie erhebt eine Reihe relevanter soziodemografischer Variablen, die sowohl als Kontrollvariablen für allgemeine Analysen als auch für spezifische sozialepidemiologische Forhigem Niveau verwendet werden sollen. Neben einer technischen Darstellung der $\mathrm{zu}$ diesem Zweck verwendeten Instrumente wurde in diesem Beitrag - zunächst theoretisch-methodisch - bewertet, ob dieser Anspruch eingelöst werden kann.

Die eingesetzten Instrumente zur Messung soziodemografischer Merkmale in der NAKO umfassen Geschlecht, Alter, Familienstand, Partnerschaft, Anzahl leiblicher Kinder, Wohnsituation, Haushaltsgröße (Zahl der Personen gesamt und 14 Jahre oder älter), Einkommen sowie die Abfrage des höchsten schulischen und beruflichen Ausbildungsabschlusses. Die Erfassung erfolgte weitgehend schung auf international konkurrenzfä- 


\section{Leitthema}

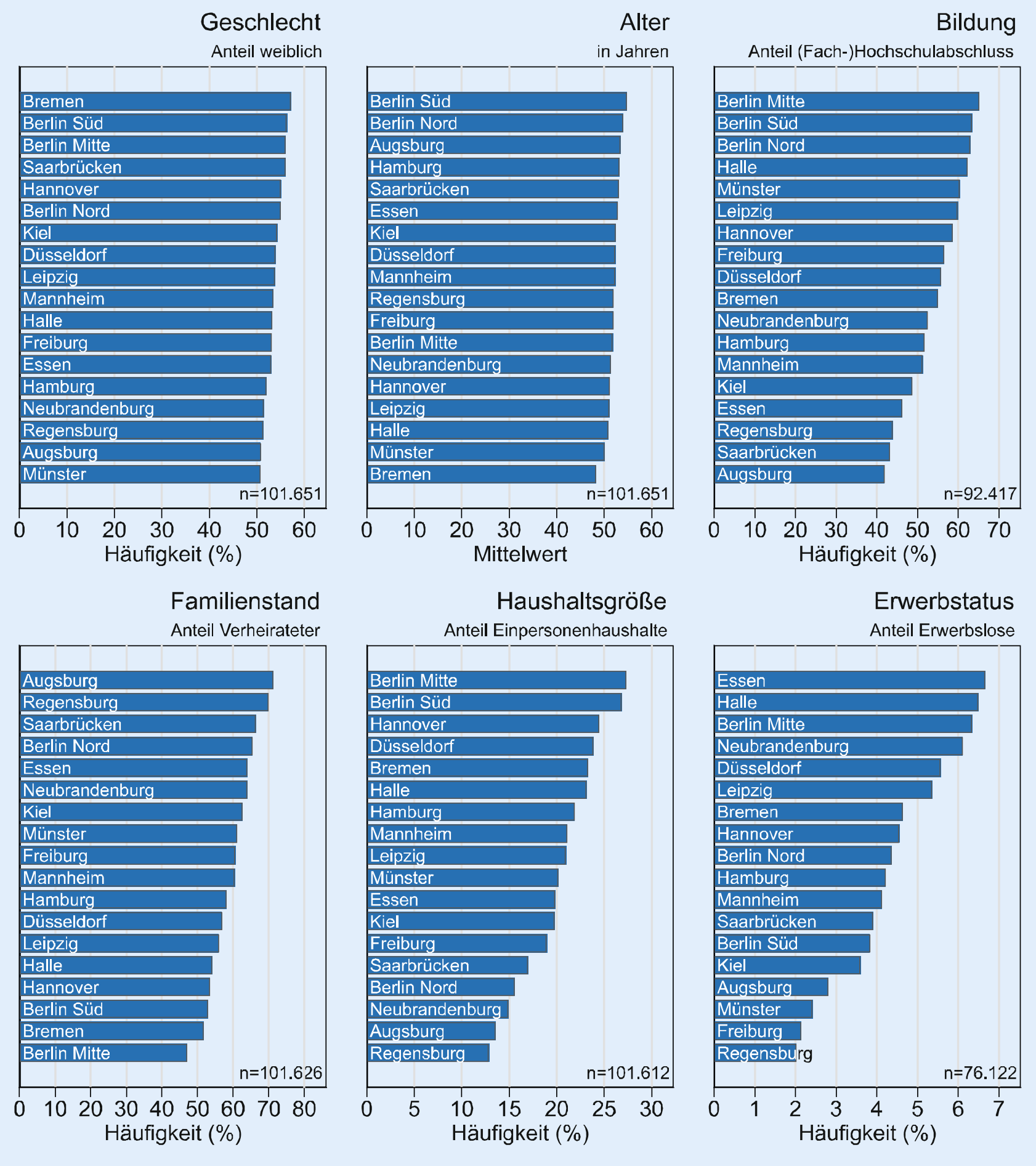

Abb. 1 \ Häufigkeiten und Lagemaße soziodemografischer und erwerbsbezogener Merkmale nach Studienzentrum. (Anteil Verheirateter umfasst nur zusammenlebende Ehepartner. Die Prozentanteile der Erwerbslosen beziehen sich auf Erwerbspersonen (Erwerbstätige und Erwerbslose ohne Nichterwerbspersonen wie Schüler, Studenten oder Rentner). (Fach-)Hochschulabschlüsse schließen auch Fachschulabschlüsse mit ein.) 
Tab. 3 Häufigkeitvon Untergewicht, Adipositas und schlechter/weniger guterselbst berichteter Gesundheit nach ausgewählten soziodemografischen und erwerbsbezogenen Merkmalen

Untergewicht

$\left(B M I<18,5 \mathrm{~kg} / \mathrm{m}^{2}\right)$

Bildung (ISCED97)

1 Grundschule

2 Haupt- und Real-

schule

3 Ausbildung

4 (Fach-)Hochschul- 115
reife

5 Fachschul- oder

abschluss

6 Promotion

Gesamt

Familienstand

Ledig

Verheiratet zusam- 364

menlebend

Verheiratet getrennt

lebend

Geschieden

Verwitwet

Gesamt

Partnerschaft

Mit (Ehe-)Partner

zusammenlebend

Mit (Ehe-)Partner

nicht zusammenle-

bend

\begin{tabular}{|c|c|c|c|c|c|c|}
\hline Ohne Partner & 293 & 1,5 & 4642 & 24,4 & 3037 & 16,8 \\
\hline Gesamt & 955 & 1,0 & 22.443 & 22,4 & 11.685 & 12,1 \\
\hline \multicolumn{7}{|l|}{ Erwerbsstatus } \\
\hline Erwerbstätig & 699 & 1,0 & 14.024 & 19,5 & 6244 & 8,9 \\
\hline Erwerbslos & 46 & 1,4 & 1067 & 31,7 & 919 & 30,1 \\
\hline Nichterwerbsperson & 202 & 0,8 & 7199 & 29,4 & 4375 & 19,1 \\
\hline Gesamt & 947 & 0,9 & 22.290 & 22,3 & 11.538 & 12,0 \\
\hline \multicolumn{7}{|c|}{ Umfang der Erwerbstätigkeit } \\
\hline $\begin{array}{l}\text { Vollzeit ( } \geq 35 \mathrm{~h} / \\
\text { Woche) }\end{array}$ & 344 & 0,7 & 9226 & 19,9 & 3609 & 8,0 \\
\hline $\begin{array}{l}\text { Teilzeit }(<35 \mathrm{~h} / \\
\text { Woche) }\end{array}$ & 324 & 1,3 & 4579 & 19,0 & 2533 & 10,8 \\
\hline Gesamt & 668 & 0,9 & 13.805 & 19,6 & 6142 & 8,9 \\
\hline \multicolumn{7}{|l|}{ Berufsstand } \\
\hline Selbstständig & 107 & 0,8 & 2578 & 20,0 & 1220 & 9,8 \\
\hline Angestellt & 739 & 0,9 & 19.446 & 23,0 & 10.234 & 12,6 \\
\hline Ausbildung & 20 & 4,0 & 67 & 13,2 & 42 & 8,6 \\
\hline Gesamt & 866 & 0,9 & 22.091 & 22,5 & 11.496 & 12,2 \\
\hline
\end{tabular}

in Übereinstimmung mit den vom Statistischen Bundesamt herausgegebenen Demographischen Standards [27] und erlaubt somit direkte Vergleiche mit dem Mikrozensus sowie mit anderen Gesundheitsstudien in Deutschland wie DEGS, CARLA, GEDA, HNR, CORA, Life-Adult und SHIP [36, 37]. Zudem ermöglicht die Erfassung der schulischen und beruflichen Bildungsabschlüsse die Ableitung der hierarchischen, international vergleichbaren ISCED-Skala [32]. Nach erfolgter Codierung der offenen Berufsabfrage wird in Zukunft auch die Bestimmung der beruflichen Stellung nach Standards wie dem EriksonGoldthorpe-Portocarero-Schema (EGP) möglich sein [38]. Ebenso ist durch die Abfrage der Haushaltszusammensetzung die Ableitung des haushaltsgewichteten Nettoäquivalenzeinkommens gegeben [28]. Letztendlich sind mit Bildung, Einkommen und beruflicher Stellung die wichtigsten Indikatoren zur Bestimmung der sozioökonomischen Position abgedeckt.

In der NAKO wurden zudem weitere Informationen zur Erwerbstätigkeit erfasst. Dazu zählen der Erwerbsstatus nach international vergleichbarem Labour-Force-Konzept [33], der Umfang der Wochenarbeitsstunden und der aktuelle bzw. zuletzt ausgeübte Beruf. Weiter existieren Module, die detailliertere Informationen zu Rente und Erwerbslosigkeit sowie zu Bildung und Beruf des Partners und der Eltern erfassen. Hierdurch bietet die NAKO auch Potenzial für die Lebenslaufepidemiologie, die sich mit Gesundheit und Krankheit in Abhängigkeit von Faktoren wie der Erwerbsbiografie oder sozialer Mobilität befasst [39].

Insgesamt ist die NAKO hinsichtlich der Erhebung soziodemografischer Merkmale nicht nur mit den bereits erwähnten Gesundheitsstudien aus Deutschland, sondern auch mit internationalen Studien ähnlichen Aufbaus wie CONSTANCES [18], LifeLines [19], CONOR [21], LifeGene [20] und der UK Biobank [22] vergleichbar. Einschränkend ist jedoch anzumerken, dass die Abfrage der Merkmale im persönlichen Interview eine Begrenzung der Fragenanzahl nötig machte, um die zeitliche 


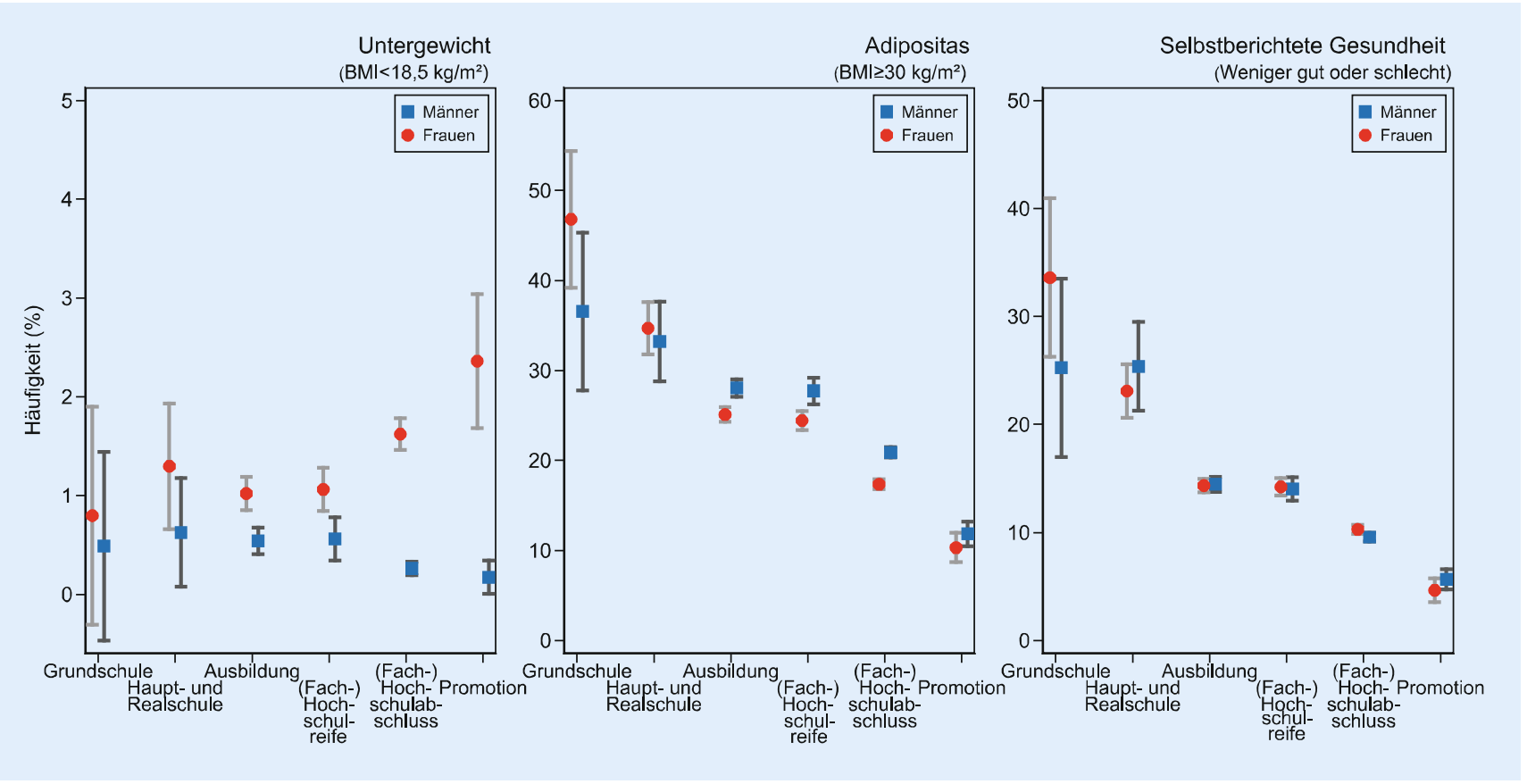

Abb. 2 ॥ Adjustierte Häufigkeiten von Untergewicht, Adipositas und schlechter selbst berichteter Gesundheit nach Bildungsstufe. (Adjustiertfür Alterund Studienzentrum. Relative Häufigkeiten und 95 \% Konfidenzintervalle. (Fach-)Hochschulabschlüsse schließen auch Fachschulabschlüsse mit ein.)

Belastung der Befragten in Grenzen zu halten. Daher ist die Tiefe der Abfrage an manchen Stellen geringer als in den Vorlagen der Demographischen Standards (z.B. fehlen eine Abfrage des Wirtschaftszweigs der aktuellen oder letzten beruflichen Tätigkeit und eine genaue Abfrage der Haushaltszusammensetzung).

Um neben der qualitativen Einordnung auch einen ersten quantitativen Eindruck von der NAKO zu erhalten, wurden Analysen mit dem Datensatz zur Halbzeit der Datenerhebung durchgeführt. Bei der Beschreibung der sozialstrukturellen Zusammensetzung der NAKO fanden wir weitgehend die erwarteten Verteilungen. So war beispielsweise der Anteil von Einpersonenhaushalten in Studienzentren mit urbaner Lage höher als bei weniger urbaner Lage. Ein Vergleich der Studienzentren zeigte zudem eine höhere Quote an Erwerbslosen in ostdeutschen Gebieten im Vergleich zu westdeutschen Standorten. Ein weiteres Beispiel ist, dass bei den Frauen ein höherer Anteil an Verwitweten, Nichterwerbstätigen und Teilzeitbeschäftigten $\mathrm{zu}$ verzeichnen war. Diese Verteilungen spiegeln bekannte regionale bzw. soziodemografische Verteilungsmuster in Deutschland wider, was als Hinweis für die Validität der Messung gewertet werden kann [30, 40, 41]. Eine systematische Prüfung der soziodemografischen Repräsentativität der Stichprobe im Vergleich zur Grundgesamtheit der in Deutschland lebenden Bevölkerung wird $\mathrm{zu}$ einem späteren Zeitpunkt erfolgen, sobald die Basiserhebung abgeschlossen ist und somit die Gesamtstichprobe verfügbar ist. Erste testweise Vergleiche des Datensatzes zur Halbzeit der Basiserhebung mit dem Mikrozensus deuten auf einen tendenziell höheren Anteil an Frauen, Höhergebildeten und Älteren in der NAKO hin [30]. Da bekannt ist, dass entsprechende soziodemografische Merkmale mit einer höheren Teilnahmebereitschaft an Gesundheitsstudien einhergehen, sollte das Ausmaß möglicher Stichprobenverzerrungen mit Vorliegen des vollständigen Datensatzes untersucht werden [42]. Eine ausführlichere Diskussion hierzu findet sich im Beitrag von Schipf et al. in diesem Themenheft.

Abschließend wurden Analysen zur Kriteriumsvalidität durchgeführt und exemplarische Untersuchungen hin- sichtlich des Zusammenhangs soziodemografischer Merkmale mit der Häufigkeit von Untergewicht, Adipositas und selbst berichteter Gesundheit angestellt. Wir fanden ausgeprägte Altersund Bildungsgradienten und konnten somit bereits bekannte Gesundheitszusammenhänge mit den Daten der NAKO replizieren [24-26]. Dies spricht für eine inhaltliche Validität der in der NAKO erhobenen soziodemografischen und erwerbsbezogenen Instrumente.

Als Fazit bleibt festzuhalten, dass mit der NAKO eine neue Ressource für die sozialepidemiologische Forschung in Deutschland entsteht. Hinsichtlich der Messung soziodemografischer und erwerbsbezogener Faktoren kann eine Detailtiefe attestiert werden, die Vergleiche mit wichtigen nationalen und internationalen bevölkerungsbasierten Kohortenstudien und die Anwendung zentraler Konzepte der Ungleichheitsforschung erlaubt. Durch die Verknüpfung dieser Konzepte mit klinischen Parametern wie Laborwerten, Bildgebungsverfahren, Körperfunktionstests und inzidenten Erkrankungen im Follow-up hat die NAKO das Potenzial, wegweisende Forschung zu ermöglichen. 


\section{Korrespondenzadresse}

\section{Prof. Dr. Nico Dragano}

Institut für Medizinische Soziologie, Centre

for Health and Society, Medizinische Fakultät, Heinrich-Heine-Universität Düsseldorf

Moorenstr. 5, 40225 Düsseldorf, Deutschland dragano@med.uni-duesseldorf.de

Danksagung. Wir möchten uns herzlich bei allen Teilnehmenden sowie den Mitarbeiterinnen und Mitarbeitern der NAKO Gesundheitsstudie bedanken.

Förderung. Dieses Projekt wurde mit Daten der NAKO Gesundheitsstudie durchgeführt (www.nako. de). Die NAKO Gesundheitsstudie wird durch das Bundesministerium für Bildung und Forschung (BMBF, Förderkennzeichen 01ER1301A/B/C und 01ER1511D), die Bundesländer und die HelmholtzGemeinschaft gefördert sowie durch die beteiligten Universitäten und Institute der Leibniz-Gemeinschaft finanziell unterstützt

Funding. Open Access funding provided by Projekt DEAL.

\section{Einhaltung ethischer Richtlinien}

Interessenkonflikt. N. Dragano, M. Reuter, H. Greiser H. Becher, H. Zeeb, R. Mikolajczyk, A. Kluttig, M. Leitzmann, B. Fischer, K.-H. Jöckel, C. Emmel, G. Krause, S. Castell, A. Damms-Machado, N. Obi, T. Schikowski, O. Kuss, W. Hoffmann, S. Schipf, T. Pischon, L. Jaeschke, L. Krist, T. Keil, W. Lieb, B. Holleczek, H. Brenner, K. Wirkner, M. Loeffler, K.B. Michels, C.-W. Franzke, A. Peters, J. Linseisen, K. Berger, N. Legath, W. Ahrens, T. Lampert und B. Schmidt geben an, dass kein Interessenkonflikt besteht.

Alle Teilnehmenden wurden ausführlich aufgeklärt und haben schriftlich ihr informiertes Einverständnis zur Studienteilnahme gegeben. Das Untersuchungsprogramm wurde im Einklang mit nationalem Recht sowie gemäß der Deklaration von Helsinki von 1975 (in der aktuellen, überarbeiteten Fassung) durchgeführt.

Open Access Dieser Artikel wird unter der Creative Commons Namensnennung 4.0 International Lizenz veröffentlicht, welche die Nutzung, Vervielfältigung, Bearbeitung, Verbreitung und Wiedergabe in jeglichem Medium und Format erlaubt, sofern Sie den/die ursprünglichen Autor(en) und die Quelle ordnungsgemäß nennen, einen Link zur Creative Commons Lizenz beifügen und angeben, ob Änderungen vorgenommen wurden.

Die in diesem Artikel enthaltenen Bilder und sonstiges Drittmaterial unterliegen ebenfalls der genannten Creative Commons Lizenz, sofern sich aus der Abbildungslegende nichts anderes ergibt. Sofern das betreffende Material nicht unter der genannten Creative Commons Lizenz steht und die betreffende Handlung nicht nach gesetzlichen Vorschriften erlaubt ist, ist für die oben aufgeführten Weiterverwendungen des Materials die Einwilligung des jeweiligen Rechteinhabers einzuholen.
Weitere Details zur Lizenz entnehmen Sie bitte der Lizenzinformation auf http://creativecommons.org/ licenses/by/4.0/deed.de.

\section{Literatur}

1. German National Cohort (GNC) Consortium (2014) The German national cohort: aims, study design and organization. Eur J Epidemiol 29(5):371-382 https://doi.org/10.1007/s10654-014-9890-7

2. WHO Commission on Social Determinants of Health (2008) Closing the gap in a generation: health equity through action on the social determinants of health. Final report of the commission on social determinants of health. World Health Organization, Geneva

3. Dahlgren G, Whitehead M (2006) European strategies for tackling social inequalities in health levelling up part 2. World Health Organisation, Copenhagen

4. Cockerham WC (2014) Medical sociology, 13. Aufl. Taylor and Francis, London

5. Richter M, Hurrelmann K (Hrsg) (2016) Soziologie von Gesundheit und Krankheit. Springer VS, Wiesbaden

6. Kolip P, Lange C, Finne E (2019) Gleichstellung der Geschlechter und Geschlechterunterschiede in der Lebenserwartung in Deutschland (Gender equality and the gender gap in life expectancy in Germany). Bundesgesundheitsblatt Gesundheitsforschung Gesundheitsschutz 62(8):943-951. https://doi.org/10.1007/s00103-019-02974-2

7. Berkman LF, Kawachi I, Glymour M (2014) Social epidemiology, 2. Aufl. Oxford University Press, Oxford

8. Kunst A, Groenhof F, Mackenbach JP (1998) Occupational class and cause specific mortality in middle aged men in 11 European countries: comparison of population based studies. BMJ 316:1636-1642

9. Niedhammer I, Bourgkard E, Chau N (2011) Occupational and behavioural factors in the explanation of social inequalities in premature and total mortality: a 12.5-year follow-up in the lorhandicap study. Eur J Epidemiol 26(1):1-12. https://doi.org/10.1007/s10654-010-9506-9

10. Mielck A (2000) Soziale Ungleichheit und Gesund heit. Empirische Ergebnisse, Erklärungsansätze, Interventionsmöglichkeiten. Huber, Bern

11. Lampert T, Kroll L (2014) Soziale Unterschiede in der Mortalität und Lebenserwartung

12. Marmot MG (2004) The status syndrome. How social standing affects our health and longevity, 1. Aufl. Times Books, New York

13. Marmot MG, Smith GD, Stansfeld S et al (1991) Health inequalities among British civil servants: the Whitehall II study. Lancet 337:1387-1393

14. Lampert T, Richter M, Schneider S, Spallek J, Dragano N (2016) Soziale Ungleichheit und Gesundheit : Stand und Perspektiven der sozialepidemiologischen Forschung in Deutschland (Social inequality and health: Status and prospect of socio-epidemiological research in Germany). Bundesgesundheitsblatt Gesundheitsforschung Gesundheitsschutz 59(2):153-165. https://doi. org/10.1007/s00103-015-2275-6

15. Lampert T, Kuntz B, Schneider S, Spallek J (2018) Soziale Ungleichheit und Gesundheit: die Entwicklung sozialepidemiologischer Forschung in Deutschland. Public Health Forum 26(3):212-215. https://doi.org/10.1515/pubhef-2018-0062
16. Geyer S (2008) Sozialstruktur und Krankheit. Analysen mit Daten der Gesetzlichen Krankenversicherung. Bundesgesundheitsblatt Gesundheitsforschung Gesundheitsschutz 51:1164-1172

17. Lampert T, Hoebel J, Kroll LE (2019) Soziale Unterschiede in der Mortalität und Lebenserwartung in Deutschland. Aktuelle Situation und Trends. J Health Monit 4:3-15. https://doi.org/10.25646/ 5868

18. Zins M, Goldberg M (2015) The French CONSTANCES population-based cohort: design, inclusion and follow-up. Eur J Epidemiol 30(12):1317-1328 https://doi.org/10.1007/s10654-015-0096-4

19. Stolk RP, Rosmalen JGM, Postma DS et al (2008) Universal risk factors for multifactorial diseases: lifelines: a three-generation population-based study. Eur J Epidemiol 23(1):67-74. https://doi. org/10.1007/s10654-007-9204-4

20. Almqvist C, Adami H-O, Franks PW et al (2011) LifeGene-a large prospective population-based study of global relevance. Eur J Epidemiol 26(1):67-77. https://doi.org/10.1007/s10654010-9521-x

21. Naess O, Søgaard AJ, Arnesen E et al (2008) Cohort profile: cohort of Norway (CONOR). Int J Epidemiol 37(3):481-485. https://doi.org/10. 1093/ije/dym217

22. Sudlow C, Gallacher J, Allen N et al (2015) UK biobank: an open access resource for identifying the causes of a wide range of complex diseases of middle and old age. PLoS Med 12(3):e1001779. https://doi.org/10.1371/journal.pmed.1001779

23. Ahrens W, Jöckel K-H (2015) Der Nutzen großer Kohortenstudien für die Gesundheitsforschung am Beispiel der Nationalen Kohorte (The benefit of large-scale cohort studies for health research: the example of the German National Cohort). Bundesgesundheitsblatt Gesundheitsforschung Gesundheitsschutz 58(8):813-821. https://doi. org/10.1007/s00103-015-2182-X

24. Kim TJ, Roesler NM, von dem Knesebeck O (2017) Causation or selection-examining the relation between education and overweight/obesity in prospective observational studies: a metaanalysis. Obes Rev 18(6):660-672. https://doi.org/ 10.1111/obr.12537

25. Schienkiewitz A, Mensink GBM, Kuhnert R, Lange C (2017) Übergewicht und Adipositas be Erwachsenen in Deutschland. J Health Monit 2(2):21-28. https://doi.org/10.17886/RKI-GBE2017-025

26. Moor I, Spallek J, Richter M (2017) Explaining socioeconomic inequalities in self-rated health: a systematic review of the relative contribution of material, psychosocial and behavioural factors. J Epidemiol Community Health 71(6):565-575. https://doi.org/10.1136/jech-2016-207589

27. Glemser A, Heckel C, von der Heyde C, HoffmeyerZlotnik JHP, Quitt H (2010) Demographische Standards. Eine gemeinsame Empfehlung des ADM Arbeitskreis Deutscher Markt- und Sozialforschungsinstitute e. V., der Arbeitsgemeinschaft Sozialwissenschaftlicher Institute e. V. (ASI) und des Statistischen Bundesamtes, 5. Aufl. Statistik und Wissenschaft, Bd. 17. DeStatis, Wiesbaden

28. Eurostat (2018) Equivalised disposable income. https://ec.europa.eu/eurostat/statisticsexplained/index.php/Glossary:Equivalised disposable_income.Zugegriffen:3.Okt. 2019

29. Lynch JW, Kaplan GA (2000) Socioeconomic factors. In: Berkman LF, Kawachi I (Hrsg) Social epidemiology. Oxford University Press, New York, S13-35 
30. Statistisches Bundesamt (2018) Bevölkerung und Erwerbstätigkeit. Haushalte und Familien. Ergebnisse des Mikrozensus 2017. Fachserie 1, Reihe 3, 2017. https://www.destatis.de/DE/ Themen/Gesellschaft-Umwelt/Bevoelkerung/ Haushalte-Familien/Publikationen/DownloadsHaushalte/haushalte-familien-2010300177004. pdf?_blob=publicationFile \&v=4. Zugegriffen: 22. Juli 2019

31. Robert-Koch-Institut (Hrsg) (2015) Gesundheit in Deutschland. Gesundheitsberichterstattung des Bundes. Robert Koch-Institut, Berlin

32. UNESCO (1997) International standard classification of education. ISCED 1997. UNESCO, Paris

33. 19. Internationale Konferenz der Arbeitsstatistiker (2014) Entschließung über Arbeitsstatistiken, Erwerbstätigkeit und die Unterauslastung des Arbeitskräfteangebots. https://www. ilo.org/wcmsp5/groups/public/---dgreports/--stat/documents/normativeinstrument/wcms_ 235273.pdf. Zugegriffen: 4. Sept. 2019

34. Mensink GBM, Schienkiewitz A, Haftenberger M, Lampert T, Ziese T, Scheidt-Nave C (2013) Übergewicht und Adipositas in Deutschland: ErgebnissederStudiezur GesundheitErwachsener in Deutschland (DEGS1) (Overweight and obesity in Germany:results of the German Health Interview and Examination Survey for Adults (DEGS1)). Bundesgesundheitsblatt Gesundheitsforschung Gesundheitsschutz 56(5-6):786-794. https://doi. org/10.1007/s00103-012-1656-3

35. WHO (2005) The SuRF report 2. Surveillance of chronic disease risk factors: country-level data and comparable estimates. World Health Organization, Geneva

36. Kurth B-M (2009) DEGS - Studie zur Gesundheit Erwachsener in Deutschland. Projektbeschreibung. Beiträge zur Gesundheitsberichterstattung des Bundes. Robert-Koch-Institut, Berlin

37. Haerting J, Kluttig A, Greiser KH, Nuding S, Werdan K (2012) Kohortenstudie zu Risikofaktoren für Herz-Kreislauf-Krankheiten in einer urbanen älteren ostdeutschen Allgemeinbevölkerung (CARLA-Studie) (A cohort study investigating risk factors for cardiovascular disease in an urban elderly East-German population (CARLA study)). Bundesgesundheitsblatt Gesundheitsforschung Gesundheitsschutz 55(6-7):795-800. https://doi. org/10.1007/s00103-012-1493-4

38. Brauns H, Haun D, Steinmann S (1997) Die Konstruktion eines international vergleichbaren Klassenschemas (EGP). Erwerbsstatistische Besonderheiten am Beispiel von Labour Force Surveys der Bundesrepublik Deutschland, Frankreichs, Großbritanniens und Ungarns. Arbeitspapiere/ Mannheimer Zentrum für Europäische Sozialforschung, Bd. 22. MZES, Mannheim

39. Kuh D, Ben-Shlomo Y (Hrsg) (2004) A life course approach to chronic disease epidemiology, 2. Aufl. Life course approach to adult health series. Oxford University Press, Oxford

40. Statistisches Bundesamt (2018) Statistisches Jahrbuch Deutschland 2018, 1. Aufl. DeStatis, Wiesbaden

41. Hammes W (2012) Haushalte und Lebensformen der Bevölkerung. Ergebnisse des Mikrozensus 2011

42. Lindsted KD, Fraser GE, Steinkohl M, Beeson WL (1996) Healthy volunteer effect in a cohort study. Temporal resolution in the adventist health study. JClin Epidemiol 49(7):783-790

43. Greiser KH, Kluttig A, Schumann B et al (2005) Cardiovascular disease, risk factors and heart rate variability in the elderlygeneral population: design and objectives of the CARdiovascular disease, living and ageing in Halle (CARLA) study. BMC Cardiovasc Disord 5:33

44. Robert Koch-Insitut (2019) GEDA 2009. https:// www.rki.de/DE/Content/Gesundheitsmonitoring/ Studien/Geda/Geda_2009_inhalt.html.Zugegriffen:3. Sept. 2019 